\title{
O uso da laserterapia de baixa intensidade na prática do enfermeiro: uma revisão integrativa
}

The use of low-level laser therapy in nursing practice: an integrative review

El uso de la terapia con láser de baja intensidad en la práctica de enfermería: una revisión integrativa

Larissa Pereira Stelet Ferreira ORCID: https://orcid.org/0000-0002-3147-5899 Universidade do Estado do Rio de Janeiro, Brasil E-mail: larissastelet15@gmail.com

Eugenio Fuentes Pérez Júnior ORCID: https://orcid.org/0000-0003-4611-0443 Universidade do Estado do Rio de Janeiro, Brasil E-mail: eugenioperezjunior@gmail.com Ariane da Silva Pires ORCID: https://orcid.org/0000-0003-1123-493X Universidade do Estado do Rio de Janeiro, Brasil E-mail: arianepiresuerj@gmail.com

Francisco Gleidson de Azevedo Gonçalves ORCID: https://orcid.org/0000-0002-6468-8137 Universidade do Estado do Rio de Janeiro, Brasil E-mail: gleydy_fran@hotmail.com

Alessandra Sant' Anna Nunes ORCID: https://orcid.org/0000-0001-7435-2568 Universidade do Estado do Rio de Janeiro, Brasil E-mail: asantnunes@gmail.com

Vânia Lima Coutinho ORCID: https://orcid.org/0000-0001-9300-3697 Universidade do Estado do Rio de Janeiro, Brasil E-mail: vlcoutto@hotmail.com

Advi Catarina Barbachan Moraes ORCID: https://orcid.org/0000-0002-1470-2623 Universidade do Estado do Rio de Janeiro, Brasil E-mail: advicbmoraes@gmail.com

Helena Ferraz Gomes

ORCID: https://orcid.org/0000-0001-6089-6361 Universidade do Estado do Rio de Janeiro, Brasil E-mail: helenafg1@yahoo.com.br

Ellen Marcia Peres

ORCID: https://orcid.org/0000-0003-4262-6987

Universidade do Estado do Rio de Janeiro, Brasil E-mail: ellenperesuerj@gmail.com

Livia Fajin de Mello

ORCID: https://orcid.org/0000-0002-5613-7976

Universidade do Estado do Rio de Janeiro, Brasil

E-mail: liviafajin@gmail.com

Priscila Cristina da Silva Thiengo de Andrade ORCID: https://orcid.org/0000-0003-0840-4838 Universidade do Estado do Rio de Janeiro, Brasil E-mail: profprithiengo@gmail.com

Carolina Cabral Pereira da Costa ORCID: https://orcid.org/0000-0002-0365-7580 Universidade do Estado do Rio de Janeiro, Brasil

E-mail: carolcuerj@hotmail.com

Carolina Gomes da Silva de Souza ORCID: https://orcid.org/0000-0002-0258-392X Universidade do Estado do Rio de Janeiro, Brasil E-mail: carolinagomescgz@gmail.com 


\section{Resumo}

Objetivo: Analisar as produções científicas acerca da utilização do laser de baixa intensidade na prática do enfermeiro. Metodologia: Trata-se de uma revisão integrativa da literatura realizada na Biblioteca Virtual em Saúde que inclui, dentre outras, as seguintes bases de dados: LILACS, MEDLINE, BDENF, IBECS, CUMED e nas bases de dados SciELO, PUBMED e Science Direct Online, entre os anos de 2016 e 2021. Resultados: Foram analisados dezesseis estudos. Em relação ao ano das publicações, evidenciou-se que dois são do ano 2016 (12,5\%); três de 2018 (18,75\%); quatro de 2019 (25\%); quatro de 2020 (25\%); três de 2021 (18,75\%). Quanto a metodologia usada nas pesquisas, observou-se que dez são revisão integrativa/sistemática/bibliográfica $(62,5 \%)$; dois são qualitativos $(12,5 \%)$ e de estudos randomizados (12,5\%); um estudo descritivo (6,25\%); um estudo metodológico $(6,25 \%)$. Identificou-se que se destaca como o uso mais frequente na prática do enfermeiro o uso do laser como terapia complementar no processo de cicatrização e reparo tecidual de lesões cutaneomucosas $(81,2 \%)$, outras utilizações do laser pelo enfermeiro observadas foram: finalidade analgésica (25\%); terapia complementar na drenagem de edemas e laser-acupuntura (18,75\%); tratamento de infecções fúngicas $(12,5 \%)$. Conclusão: Nos últimos cinco anos foi possível identificar a escassez da produção científica na área. A produção encontrada nas bases de dados nacionais e internacionais são incipientes e apresentam métodos baseados em revisões e poucos estudos clínicos de alto rigor metodológico.
\end{abstract}

Palavras-chave: Terapia com luz de baixa intensidade; Onicomicose; Enfermagem; Podiatria; Diabetes Mellitus.

\begin{abstract}
Objective: To analyze scientific productions about the use of lasers in nursing practice. Methodology: This is an integrative literature review carried out in the Virtual Health Library that includes, among others, the following databases: LILACS, MEDLINE, BDENF, IBECS, CUMED and in the SciELO, PUBMED and Science Direct Online databases, among the years 2016 and 2021. Results: Sixteen studies were analyzed. Regarding the year of publications, it was evidenced that two (02) are from the year 2016 (12.5\%); three (03) from 2018 (18.75\%); four (04) from 2019 (25\%); four (04) of 2020 (25\%); three (03) from 2021 (18.75\%). Regarding the methodology used in the researches, it was observed that ten (10) are integrative/systematic/bibliographic review (62.5\%); two (02) are qualitative (12.5\%) and from randomized studies $(12.5 \%)$; one (01) descriptive study $(6.25 \%)$ and one $(01)$ methodological study $(6.25 \%)$. It was identified that the most frequent use in nursing practice is the use of laser as a complementary therapy in the healing process and tissue repair of cutaneomucosal lesions (81.2\%), other uses of laser by the nurse observed were: analgesic purpose (25\%); complementary therapy in the drainage of edemas and laser acupuncture (18.75\%); treatment of fungal infections $(12.5 \%)$. Conclusion: In the last five years, it was possible to identify the scarcity of scientific production in the area. The production found in national and international databases is incipient and presents methods based on reviews and few clinical studies of high methodological rigor.
\end{abstract}

Keywords: Low-level light therapy; Onychomycosis; Nursing; Podiatry; Diabetes Mellitus.

\title{
Resumen
}

Objetivo: Analizar las producciones científicas sobre el uso de láseres en la práctica de enfermería. Metodología: Se trata de una revisión integrativa de la literatura realizada en la Biblioteca Virtual en Salud que incluye, entre otras, las siguientes bases de datos: LILACS, MEDLINE, BDENF, IBECS, CUMED y en las bases de datos SciELO, PUBMED y Science Direct Online, entre 2016 y 2021. Resultados: Se analizaron dieciséis estudios. En relación al año de las publicaciones, se evidenció que dos (02) son del año 2016 (12,5\%); tres (03) de 2018 (18,75\%); cuatro (04) de 2019 (25\%); cuatro (04) de 2020 (25\%); tres (03) de 2021 (18,75\%). En cuanto a la metodología utilizada en las investigaciones, se observó que diez (10) son revisión integrativa/sistemática/bibliográfica $(62,5 \%)$; dos $(02)$ son cualitativos $(12,5 \%)$ y de estudios aleatorizados (12,5\%); un (01) estudio descriptivo $(6,25 \%)$ y un (01) estudio metodológico $(6,25 \%)$. Se identificó que se destaca como el uso más frecuente en la práctica del enfermero el uso del láser como terapia complementaria en el proceso de cicatrización y reparación tisular de lesiones cutaneomucosas $(81,2 \%)$, otros usos del láser por el enfermero observados fueron: finalidad analgésica $(25 \%)$; terapia complementaria en el drenaje de edemas y láser-acupuntura (18,75\%); tratamiento de infecciones fúngicas (12,5\%). Conclusión: En los últimos cinco años se pudo identificar la escasez de producción científica en la zona. La producción encontrada en bases de datos nacionales e internacionales es incipiente y presenta métodos basados en revisiones y escasos estudios clínicos de alto rigor metodológico.

Palabras clave: Terapia por luz de baja intensidad; Onicomicosis; Enfermería; Podiatría; Diabetes Mellitus.

\section{Introdução}

A aplicação do laser de baixa intensidade apresenta uso crescente na prática clínica de diversos profissionais de saúde, seus recursos terapêuticos vêm sendo amplamente utilizados graças a seus efeitos como: melhoria da qualidade da cicatrização, estímulo a microcirculação, efeitos anti-inflamatórios, antiedematosos, analgésicos e no controle de infecções (Oliveira et al., 2021). 
O termo laser, é um acrônimo da expressão em inglês Light Amplification by Stimulated Emission of Radiation, que significa a luz amplificada com a emissão estimulada de radiação. Como características físicas, a luz do laser é uma radiação eletromagnética, unidirecional e monocromática capaz de transmitir pacotes de energia denominado fótons (Gomes \& Schapochnick, 2017).

As propriedades terapêuticas do laser começaram a ser estudadas por Einstein desde 1917, com a proposição da teoria da emissão. A partir de 1960, com a construção do primeiro emissor de laser a Rubi por Theodore Maiman, o laser começou a ser usado como instrumento terapêutico (Henriques et al., 2010). O laser pode ser classificado em alta potência e baixa potência, o primeiro emite radiação ionizante que resulta em alteração no tecido, atua na remoção, corte e coagulação dos tecidos, enquanto o segundo, laser de baixa potência, emite radiação não ionizante e atua no processo de reparação tecidual e celular, promove alívio da dor, drenagem de edemas, controla infecções nos tecidos e controla o processo inflamatório (Júnior et al., 2021).

Ao considerar seus efeitos terapêuticos a Laserterapia de Baixa Intensidade (LBI) configura-se como um importante recurso incorporado a prática clínica de enfermagem. Sua utilização deve ser realizada pelo enfermeiro devidamente capacitado, uma vez que o uso da LBI requer do profissional conhecimentos em física, interação laser e tecido biológico, dosimetria, biofotônica, além da fisiologia e reabilitação, e inserida na Sistematização da Assistência de Enfermagem (Conselho Regional de Enfermagem de São Paulo, 2014).

A aplicação deste laser vem crescendo cada vez mais, sendo uma área de grande interesse dos enfermeiros, devido os efeitos biomoduladores, principalmente na reparação tecidual, sendo utilizado preventivamente, bioestimulando a reparação em feridas cirúrgicas ou ainda bioinibindo processos inflamatórios e infecciosos já instalados em feridas crônicas (COREN-SP, 2014).

O LBI acelera o processo regenerativo e a qualidade do tecido formado, atuando na sequência de eventos fisiológicos e bioquímicos decorrentes do processo, como a inflamação, a síntese de colágeno, a formação do tecido de granulação e a reepitelização. A energia absorvida é transformada em adenosina trifosfato e usada pela célula para atividades metabólicas, como proliferação celular, síntese de colágeno e aceleração do processo de reparação do tecido em animais e humanos. O LBI não possui potencial destrutivo (Busatta et al., 2018).

A LBI também tem aplicabilidade na fotoinativação microbiana produzida pela terapia fotodinâmica. O LBI, ao contrário dos lasers de alta, não tem a capacidade de descontaminação, por não provocarem aumento de temperatura no tecido. O efeito antimicrobiano é alcançado quando utilizado em associação com agentes fotossensibilizadores, através da terapia fotodinâmica, ocorre a produção de espécies reativas de oxigênio capazes de inativar vírus, bactérias e fungos (Bernardes \& Jurado, 2018).

Devido à escassez de publicações na área de enfermagem acerca da atuação do enfermeiro na área de Laserterapia, o estudo em tela mostra-se relevante e contribuirá com a ampliação do conhecimento sobre essa temática e o desenvolvimento de mais estudos na área. Diante desse contexto, o presente estudo tem como objetivo analisar as produções científicas acerca da utilização do laser de baixa intensidade na prática do enfermeiro.

\section{Metodologia}

Trata-se de uma revisão integrativa de literatura, método que tem por objetivo realizar sobre o conhecimento existente em pesquisas sobre um tema, possibilitando uma síntese de vários estudos com metodologias diferentes (Botelho et al., 2011), sobre as produções científicas acerca do uso do laser de baixa intensidade pelos enfermeiros. Foram adotadas as seguintes etapas: elaboração da questão norteadora; busca na literatura e definição de critérios para inclusão e exclusão de estudos; coleta de dados; análise dos estudos incluídos na revisão integrativa; interpretação dos resultados e a apresentação da revisão integrativa. 
Os artigos foram capturados por meio de busca bibliográfica, no período de maio a junho de 2021. Para a elaboração da questão norteadora, utilizou-se a estratégia PICo, definindo-se: P = população: “estudos publicados”, I = interesse: “uso do laser” e Co = contexto: "prática de enfermagem". Portanto, a questão deste estudo foi: O que há de publicação científica sobre a utilização do LBI na prática do enfermeiro? Utilizou-se a Biblioteca Virtual em Saúde (BVS) que inclui, dentre outras, as seguintes bases de dados: Literatura Latino-Americana e do Caribe em Ciências da Saúde (LILACS); Medical Literature Analysis and Retrieval System Online (MEDLINE); Base de Dados Específica da Enfermagem (BDENF); Índice Bibliográfico Espanhol de Ciências da Saúde (IBECS); Centro Nacional de Informação de Ciências Médicas de Cuba (CUMED); Scientific Eletronic Library Online (SCIELO); PubMed - U.S. National Library of Medicine e Science Direct Online.

Foram utilizados na busca os descritores em saúde cadastrados na biblioteca Decs/Mesh: "Enfermagem e Terapia com Luz de Baixa Intensidade"; "Enfermagem e lasers”, bem como sua tradução para o inglês e espanhol. Foram incluídos artigos publicados no período de 2016 a 2021, disponíveis em textos completos, nas versões português, inglês e espanhol e que respondessem à questão norteadora da pesquisa. Foram excluídos estudos duplicados, estudos publicados apenas como resumo, teses, dissertações, relatos de casos e estudos de revisão narrativa e integrativa.

Na busca inicial, encontrou-se um total de 1.539 publicações que atendiam aos descritores e aos critérios de inclusão. Através da leitura do título e resumo foi possível excluir as publicações que não estão disponíveis em texto completo, estudos não finalizados e estudos publicados apenas como resumo, teses, dissertações, relatos de casos e estudos de revisão narrativa.

Dessa forma, foram selecionados 34 estudos para leitura na íntegra. Destes, 18 não foram selecionados por se tratar de publicações em duplicidade nas diferentes bases de dados e por não responderem à questão norteadora. Dessa forma, dos 34 manuscritos lidos na íntegra, apenas 16 farão parte da amostra final desta revisão. A Figura 1 descreve a trajetória realizada para a identificação, triagem, elegibilidade e amostra final do estudo, seguindo os critérios do PRISMA.

Figura 1. Fluxograma da seleção dos estudos nas bases de dados selecionadas seguindo os critérios do PRISMA

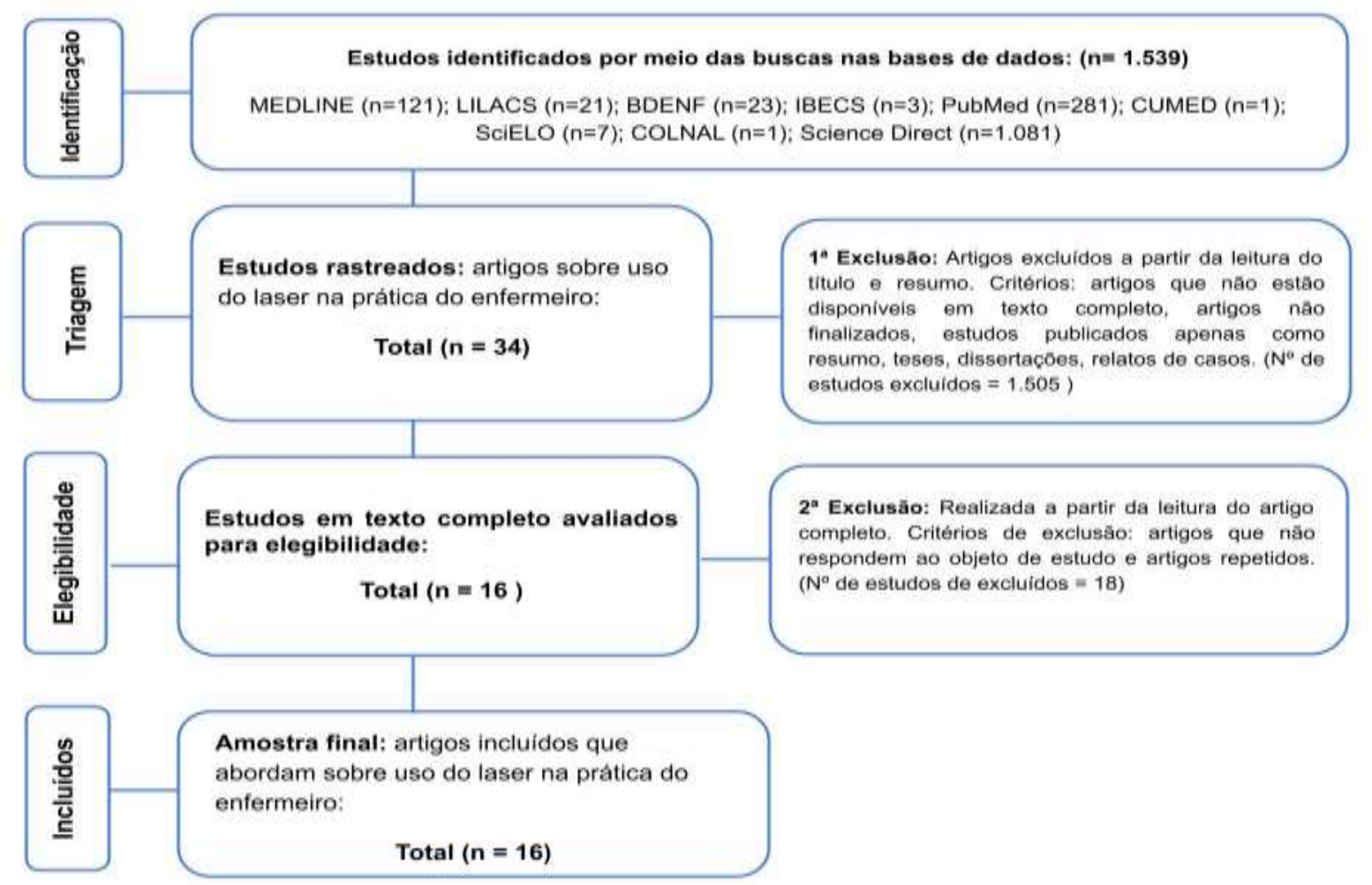

Fonte: Autores (2021). 
Para análise dos estudos incluídos, organizou-se um quadro com o conteúdo de cada publicação de acordo com os seguintes itens: título, ano de publicação, revista, tipo de estudo, amostra, objetivo, nível de evidência e o que o artigo aborda em relação à questão norteadora. A análise do nível de evidência foi realizada a partir dos conceitos propostos por Melnyk e Fineout-Overholt (2014): nível I - evidências oriundas de revisão sistemática ou metanálise de todos os ensaios clínicos relevantes randomizados controlados ou provenientes de diretrizes clínicas baseadas em revisões sistemáticas de ensaios clínicos randomizados controlados; nível II - evidências derivadas de pelo menos um ensaio clínico randomizado controlado bem delineado; nível III - evidências obtidas de ensaios clínicos bem delineados sem randomização; nível IV - evidências provenientes de estudos de coorte e de caso-controle bem delineados; nível V - evidências originárias de revisão sistemática de estudos descritivos e qualitativos; nível VI - evidências derivadas de um único estudo descritivo ou qualitativo; e nível VII evidências oriundas de opinião de autoridades e/ou relatórios de comitês de especialistas.

A partir deste quadro, foi realizada a interpretação dos resultados e apresentação da revisão integrativa.

\section{Resultados}

No período estudado (2016 a 2021), foram identificados dezesseis artigos científicos que atenderam aos critérios de inclusão (Quadro 1).

Quadro 1. Caracterização das produções científicas de acordo com título, ano, revista, tipo de estudo, amostra, objetivo e principais resultados.

\begin{tabular}{|c|c|c|c|c|}
\hline Título & $\begin{array}{c}\text { Autores/ } \\
\text { Periódico/ } \\
\text { Ano }\end{array}$ & $\begin{array}{l}\text { Estudo, Amostra e } \\
\text { Nível de Evidência }\end{array}$ & Objetivo & $\begin{array}{c}\text { Utilização do laser pelo } \\
\text { enfermeiro }\end{array}$ \\
\hline $\begin{array}{l}\text { 1.Uso do laser de baixa } \\
\text { intensidade nas } \\
\text { radiodermites: revisão } \\
\text { sistemática }\end{array}$ & $\begin{array}{l}\text { Rodrigues JMS, } \\
\text { Acosta AS, } \\
\text { Gouvea PB, } \\
\text { Massaroli R., } \\
\text { J. nurs. Health } \\
2020\end{array}$ & $\begin{array}{l}\text { Revisão sistemática. } \\
\text { Amostra: } 7 \\
\text { NE: I }\end{array}$ & $\begin{array}{l}\text { Analisar e categorizar o método de } \\
\text { uso e benefícios e principais } \\
\text { considerações sobre uso do laser em } \\
\text { lesões induzidas por radiação. }\end{array}$ & $\begin{array}{c}\text { Cicatrização e reparo } \\
\text { tecidual }\end{array}$ \\
\hline $\begin{array}{c}\text { 2.Laserterapia de baixa } \\
\text { intensidade no tratamento } \\
\text { de feridas e a atuação da } \\
\text { enfermagem }\end{array}$ & $\begin{array}{l}\text { Lima NEP, et al. } \\
\text { Rev Enferm UFPI } \\
2018\end{array}$ & $\begin{array}{l}\text { Revisão integrativa. } \\
\text { Amostra: } 11 \\
\text { NE:VI }\end{array}$ & $\begin{array}{c}\text { Identificar os benefícios da } \\
\text { laserterapia no tratamento de feridas e } \\
\text { a atuação da enfermagem neste } \\
\text { processo. }\end{array}$ & $\begin{array}{l}\text { Cicatrização e reparo } \\
\text { tecidual, Analgesia, } \\
\text { Drenagem de edema }\end{array}$ \\
\hline $\begin{array}{l}\text { 3.O uso do laser de baixa } \\
\text { potência por enfermeiro } \\
\text { no tratamento de lesões } \\
\text { cutâneas e orais. }\end{array}$ & $\begin{array}{l}\text { Armelin MVAL, } \\
\text { et al. } \\
\text { Revista Nursing } \\
2019\end{array}$ & $\begin{array}{l}\text { Revisão integrativa. } \\
\text { Amostra: } 4 \\
\text { NE:VI }\end{array}$ & $\begin{array}{l}\text { Verificar a produção científica } \\
\text { nacional e internacional acerca da } \\
\text { utilização da laserterapia pelo } \\
\text { enfermeiro no tratamento de lesões } \\
\text { cutâneas e orais }\end{array}$ & $\begin{array}{c}\text { Cicatrização e reparo } \\
\text { tecidual }\end{array}$ \\
\hline $\begin{array}{l}\text { 4.Prevenção e tratamento } \\
\text { da Mucosite em } \\
\text { ambulatório de } \\
\text { Oncologia: uma } \\
\text { construção coletiva }\end{array}$ & $\begin{array}{l}\text { Lopes LD, et al. } \\
\text { Texto Contexto } \\
\text { Enferm } \\
2016\end{array}$ & $\begin{array}{l}\text { Pesquisa } \\
\text { Convergente- } \\
\text { Assistencial } \\
\text { Amostra: } 6 \\
\text { NE: VI }\end{array}$ & $\begin{array}{c}\text { Elaborar um protocolo assistencial de } \\
\text { enfermagem para prevenção e } \\
\text { tratamento da mucosite induzida por } \\
\text { quimioterapia }\end{array}$ & $\begin{array}{c}\text { Cicatrização e reparo } \\
\text { tecidual }\end{array}$ \\
\hline $\begin{array}{l}\text { 5. Validação de um } \\
\text { protocolo de } \\
\text { auriculoterapia com laser } \\
\text { para dor crônica na coluna } \\
\text { vertebral } \\
\end{array}$ & $\begin{array}{l}\text { Nassif MS, et al. } \\
\text { Rev Min Enferm } \\
2020\end{array}$ & $\begin{array}{c}\text { Estudo } \\
\text { metodológico } \\
\text { Amostra: } 13 \text { artigos } \\
\text { e } 20 \text { voluntários. } \\
\text { NE: I } \\
\end{array}$ & $\begin{array}{l}\text { Validar um protocolo de } \\
\text { auriculoterapia com laser para dor } \\
\text { crônica na coluna vertebral. }\end{array}$ & $\begin{array}{c}\text { Laser-acupuntura } \\
\text { Analgesia }\end{array}$ \\
\hline $\begin{array}{l}\text { 6.Protocolo de laser- } \\
\text { acupuntura para } \\
\text { hipertensão arterial } \\
\text { sistêmica primária: ensaio } \\
\text { clínico randomizado }\end{array}$ & $\begin{array}{l}\text { Pereira RDM, et } \\
\text { al. } \\
\text { Rev. Latino-Am. } \\
\text { Enfermagem } \\
2018\end{array}$ & $\begin{array}{l}\text { Ensaio clínico } \\
\text { randomizado, } \\
\text { multicêntrico, } \\
\text { triplo-cego, dois } \\
\text { braços. } \\
\text { Amostra: } 102 \\
\text { NE: II }\end{array}$ & $\begin{array}{l}\text { Avaliar a eficácia de um protocolo } \\
\text { para laser-acupuntura, desenvolvido e } \\
\text { aplicado por enfermeiros em } \\
\text { pacientes com hipertensão arterial. }\end{array}$ & Laser-acupuntura \\
\hline
\end{tabular}




\begin{tabular}{|c|c|c|c|c|}
\hline $\begin{array}{l}\text { 7.Ação da terapia a laser } \\
\text { de baixa intensidade na } \\
\text { cicatrização de ulcerações } \\
\text { diabéticas }\end{array}$ & $\begin{array}{c}\text { Castro MF de, } \\
\text { Barbosa LRP, } \\
\text { Silva LL da. } \\
\text { Research, Society } \\
\text { and Development } \\
2020\end{array}$ & $\begin{array}{l}\text { Revisão integrativa } \\
\text { Amostra: } 10 \\
\text { NE:VI }\end{array}$ & $\begin{array}{c}\text { Verificar evidências acerca da } \\
\text { eficácia da terapia a laser de baixa } \\
\text { intensidade na reparação tecidual de } \\
\text { úlceras diabéticas dos pés. }\end{array}$ & $\begin{array}{c}\text { Cicatrização e reparo } \\
\text { tecidual }\end{array}$ \\
\hline $\begin{array}{l}\text { 8.Efeitos da laserterapia } \\
\text { no tratamento de lesões } \\
\text { por pressão: uma revisão } \\
\text { sistemática } \\
\end{array}$ & $\begin{array}{l}\text { Bernardes LO, } \\
\text { Jurado SR. } \\
\text { Revista Cuidarte } \\
2018 \\
\end{array}$ & $\begin{array}{l}\text { Revisão sistemática } \\
\text { Amostra: } 11 \\
\text { NE: I }\end{array}$ & $\begin{array}{l}\text { Estudar a eficácia da laserterapia no } \\
\text { processo de cicatrização de lesões por } \\
\text { pressão. }\end{array}$ & $\begin{array}{c}\text { Cicatrização e reparo } \\
\text { tecidual }\end{array}$ \\
\hline $\begin{array}{c}\text { 9.Implementação do } \\
\text { serviço de enfermagem } \\
\text { em Podiatria Clínica em } \\
\text { unidade pública de saúde } \\
\text { ambulatorial }\end{array}$ & $\begin{array}{l}\text { Pires, A. da S., et } \\
\text { al. } \\
\text { Research, Society } \\
\text { and Development } \\
2021\end{array}$ & $\begin{array}{c}\text { Pesquisa } \\
\text { Convergente } \\
\text { Assistencial } \\
\text { Amostra: } 1 \\
\text { NE: VI }\end{array}$ & $\begin{array}{l}\text { Descrever a implementação de } \\
\text { serviço de Enfermagem em Podiatria } \\
\text { Clínica em unidade pública de saúde } \\
\text { do Estado do Rio de Janeiro. }\end{array}$ & $\begin{array}{c}\text { Cicatrização e reparo } \\
\text { tecidual, Tratamento } \\
\text { infecção fúngica, Analgesia } \\
\text { Drenagem de edema }\end{array}$ \\
\hline $\begin{array}{l}\text { 10.Intervenções de } \\
\text { enfermagem para } \\
\text { pacientes com lesão por } \\
\text { pressão }\end{array}$ & $\begin{array}{l}\text { Oliveira DMN, } \\
\text { Costa MML, } \\
\text { Malagutti W. } \\
\text { Rev enferm UFPE } \\
\text { online } \\
2019 \\
\end{array}$ & $\begin{array}{l}\text { Revisão integrativa } \\
\text { Amostra: } 31 \\
\text { NE:VI }\end{array}$ & $\begin{array}{c}\text { Analisar a produção científica acerca } \\
\text { das intervenções de enfermagem da } \\
\text { CIPE para pacientes com lesão por } \\
\text { pressão. }\end{array}$ & $\begin{array}{c}\text { Cicatrização e reparo } \\
\text { tecidual }\end{array}$ \\
\hline $\begin{array}{l}\text { 11.Laser-acupuntura no } \\
\text { controle da glicemia na } \\
\text { diabetes tipo II: ensaio } \\
\text { clínico randomizado }\end{array}$ & $\begin{array}{l}\text { Pereira CD, et al. } \\
\text { Enfermagem } \\
\text { Global } \\
2021\end{array}$ & $\begin{array}{l}\text { Ensaio clínico } \\
\text { randomizado, triplo- } \\
\text { cego } \\
\text { Amostra: } 42 \\
\text { NE: II } \\
\end{array}$ & $\begin{array}{c}\text { Avaliar a eficácia da acupuntura a } \\
\text { laser aplicada à assistência de } \\
\text { enfermagem a pessoas com diabetes } \\
\text { mellitus tipo II. }\end{array}$ & Laser-acupuntura \\
\hline $\begin{array}{l}\text { 12.Laserterapia: a } \\
\text { utilização da tecnologia } \\
\text { na intervenção em } \\
\text { enfermagem }\end{array}$ & $\begin{array}{l}\text { Schmidt MH, } \\
\text { Pereira AD. } \\
\text { Disciplinarum } \\
\text { Scientia } \\
2016 \\
\end{array}$ & $\begin{array}{c}\text { Revisão } \\
\text { bibliográfica } \\
\text { Amostra: } 1 \\
\text { NE:VI }\end{array}$ & $\begin{array}{c}\text { Verificar a produção científica acerca } \\
\text { da utilização do laser pelo enfermeiro } \\
\text { no tratamento de feridas nos últimos } \\
\text { cinco anos. }\end{array}$ & $\begin{array}{c}\text { Cicatrização e reparo } \\
\text { tecidual }\end{array}$ \\
\hline $\begin{array}{l}\text { 13.Laserterapia de Baixa } \\
\text { Intensidade no tratamento } \\
\text { adjuvante em lesões } \\
\text { cutâneas: revisão } \\
\text { bibliográfica } \\
\end{array}$ & $\begin{array}{l}\text { Persilva M. } \\
\text { Revista Feridas } \\
2019\end{array}$ & $\begin{array}{l}\text { Revisão } \\
\text { bibliográfica } \\
\text { Amostra: } \\
\text { NE:VI }\end{array}$ & $\begin{array}{l}\text { Descrever os efeitos, o mecanismo de } \\
\text { ação, parâmetros mais adequados e } \\
\text { possíveis benefícios do uso do laser } \\
\text { terapêutico na cicatrização de lesões } \\
\text { cutâneas. }\end{array}$ & $\begin{array}{c}\text { Cicatrização e reparo } \\
\text { tecidual }\end{array}$ \\
\hline $\begin{array}{l}\text { 14.Laserterapia de Baixa } \\
\text { Intensidade: } \\
\text { características dos clientes } \\
\text { atendidos no serviço de } \\
\text { Podiatria }\end{array}$ & $\begin{array}{l}\text { Pérez Júnior EF, } \\
\text { et al. } \\
\text { Research, Society } \\
\text { and Development } \\
2021\end{array}$ & $\begin{array}{l}\text { Estudo descritivo, } \\
\text { retrospectivo e } \\
\text { documental } \\
\text { Amostra: } 110 \\
\text { prontuários } \\
\text { NE: VI }\end{array}$ & $\begin{array}{l}\text { Descrever e analisar as características } \\
\text { dos clientes submetidos a laserterapia } \\
\text { de baixa intensidade acompanhados } \\
\text { em um serviço de Podiatria Clínica. }\end{array}$ & $\begin{array}{l}\text { Cicatrização e reparo } \\
\text { tecidual } \\
\text { Tratamento infecção } \\
\text { fúngica } \\
\text { Analgesia } \\
\text { Drenagem de edema }\end{array}$ \\
\hline $\begin{array}{l}\text { 15. Terapia a laser de } \\
\text { baixa potência no } \\
\text { tratamento de feridas }\end{array}$ & $\begin{array}{l}\text { Bavaresco T, et } \\
\text { al. } \\
\text { Rev enferm UFPE } \\
\text { on line. } \\
2019\end{array}$ & $\begin{array}{l}\text { Revisão integrativa } \\
\text { Amostra: } 24 \\
\text { NE:VI }\end{array}$ & $\begin{array}{c}\text { Identificar a ação da terapia a laser de } \\
\text { baixa potência na cicatrização de } \\
\text { feridas. }\end{array}$ & $\begin{array}{c}\text { Cicatrização e reparo } \\
\text { tecidual }\end{array}$ \\
\hline $\begin{array}{l}\text { 16.Efeitos da laserterapia } \\
\text { de baixa intensidade na } \\
\text { cicatrização de úlceras } \\
\text { nos pés em pessoas com } \\
\text { diabetes mellitus }\end{array}$ & $\begin{array}{l}\text { Brandão MGSA, } \\
\text { et al. } \\
\text { Revista Estigma } \\
2020\end{array}$ & $\begin{array}{l}\text { Revisão integrativa } \\
\text { Amostra: } 06 \\
\text { NE:VI }\end{array}$ & $\begin{array}{l}\text { Identificar os efeitos da laserterapia } \\
\text { de baixa intensidade na cicatrização } \\
\text { do pé diabético. }\end{array}$ & $\begin{array}{l}\text { Cicatrização e reparo } \\
\text { tecidual }\end{array}$ \\
\hline
\end{tabular}

Fonte: Autores (2021).

Em relação ao ano das publicações, evidenciou-se que dois (02) são do ano 2016 (12,5\%); três (03) de 2018 (18,75\%); quatro (04) de 2019 (25\%); quatro (04) de 2020 (25\%); três (03) de 2021 (18,75\%).

Quanto ao método adotado nas pesquisas, observou-se que dez (10) são revisão integrativa/sistemática/bibliográfica (62,5\%); dois (02) são qualitativos (12,5\%) e de estudos randomizados (12,5\%); um (01) estudo descritivo (6,25\%) e um (01) estudo metodológico $(6,25 \%)$.

Quanto ao nível de evidência (NI), notou-se que onze (11) possuem NI VI (68,75\%); três (03) com NI I (18,75\%); dois (02) com NI II (12,5\%). 
Em relação ao que há publicado sobre a utilização do LBI na prática do enfermeiro, nesse estudo, identificou-se que o seu uso mais frequente como terapia complementar, realiza-se no processo de cicatrização e reparo tecidual de lesões cutaneomucosas, descrita em 81,2\% dos artigos selecionados. $25 \%$ das produções científicas selecionadas descrevem o uso com finalidade analgésica. $18,75 \%$ destacam utilização na drenagem de edemas e laser-acupuntura e 12,5\% relatam no tratamento de infecções fúngicas (dermatofitoses).

\section{Discussão}

A partir dos resultados obtidos na pesquisa, revela-se o pequeno quantitativo de artigos publicados no período estudado (2016 a 2021), o que reflete a pouca quantidade de pesquisas produzidas acerca dessa temática na área de enfermagem. Grande parte das publicações encontradas durante a etapa de busca nas bases de dados descrevem o uso do laser em outras áreas como odontologia, fisioterapia e medicina, o que indica a baixa produção de conhecimento sobre LBI na práxis do enfermeiro.

A limitação de publicações sobre o tema dificulta a ampliação de conhecimento sobre o uso do LBI como uma tecnologia associada ao cuidado de enfermagem como tratamento complementar, não invasivo, de baixo custo e sem eventos adversos. As dificuldades relacionadas a influência do sistema capitalista na produção conhecimento em enfermagem que orientam para produções quantitativas e de alto impacto, que exigem investimentos em suporte logístico de pesquisa cada vez mais escassos, constituem-se em obstáculos a serem superados (Lino et al., 2017).

Quanto aos métodos utilizados nas pesquisas em enfermagem relacionadas ao LBI relaciona-se diretamente a qualidade dos estudos e a produção de evidências cientificas capazes de impactar na realidade da prática. Ressalta-se a predominância de publicações de revisão integrativa, o que demonstra uma escassez de realização de pesquisa de campo, e/ou pesquisas clínicas que são capazes de produzir evidência científicas de alto valor para embasar a prática de enfermagem.

Dentre os fatores que contribuem para dificultar as produções cita-se o bloqueio da criatividade a prática da repetição relacionados ao critério de produtividade que impõe $\mathrm{m}$ a necessidade de muitas produções em curto prazo, impulsionando pesquisadores a publicar de qualquer modo e incentivando a pesquisas que ignoram relevantes problemas no cotidiano em saúde, que agregam baixo interesse no ponto de vista prático (Lino et al., 2017).

Por consequência da baixa quantidade de estudos que apresentam níveis de evidência I e II que apresenta qualidade dos dados científicos disponível e que define a confiança na informação possibilitando a recomendação de sua adoção na prática. A Prática Baseada em Evidências (PBE) é uma estratégia usada para que as melhores evidências científicas possam ser utilizadas na prática profissional. A PBE tem como delineamento de melhor evidência, os ensaios clínicos controlados e randomizados (Danski et al., 2017).

Nos resultados obtidos, é possível perceber que a maior parte dos estudos são revisões integrativas e revisões sistemáticas, com isso, é possível perceber que os profissionais de enfermagem possuem poucas evidências disponíveis, uma vez que as publicações de pesquisas clínicas que tem melhor nível de evidência por serem realizadas com o objetivo de aprimorar medidas de prevenção, diagnósticos e tratamentos ao paciente, são menos realizadas.

As pesquisas são imprescindíveis para que os profissionais de enfermagem possam realizar um cuidado de qualidade e cada vez mais melhorar a assistência aos pacientes através da criação de estudos inovadores na área, (Reichembach \& Pontes, 2020). Assim, observa-se a importância da ampliação de produção de pesquisas clínicas sobre o uso da LBI como instrumento de aprimoramento na prática de enfermagem e na formação de profissionais com visão crítica e conhecimento científico, a fim de capacitar enfermeiros no desenvolvimento do cuidado ao paciente baseado em evidências científicas (Pedrolo et al., 2009).

Ressalta-se que o desenvolvimento de pesquisas científicas e de desenvolvimento tecnológico acontece, em maior número, nas universidades públicas do país, o que significa que são realizadas por docentes e discentes no âmbito dos programas 
de graduação, extensão, pós-graduação, mestrado e doutorado, responsáveis por uma parcela significativa da produção científica nacional. A falta de investimentos em educação e pesquisa, como a adoção de políticas de corte de recursos na área, representam o fator de maior impacto em relação ao atraso do crescimento científico do país (Almeida et al, 2020).

Quanto ao uso do LBI na prática do enfermeiro a cicatrização de feridas é a principal indicação verificada, fato que pode estar relacionado ao fato de ser o tratamento de feridas uma atividade desenvolvida por esse profissional. O processo de cicatrização é complexo, e o uso do LBI tem tido resultados significativos nesse processo (Bavaresco et al., 2019).

A LBI tem se mostrado um recurso terapêutico eficaz na promoção da recuperação de tecidos, favorecendo o processo de cicatrização, estimulando a formação da microcirculação, por seus efeitos antinflamatórios, antiedematosos e analgésicos (Macedo et al., 2021).

Outras indicações para o uso da LBI como o tratamento de dermatofitoses, analgesia, drenagem de edemas, e ação antiinflamatória sistêmica embora possuam várias evidências científicas que corroboram sua utilização, ainda carecem de investigações na área de enfermagem.

\section{Conclusão}

Ao se analisar as produções científicas acerca da utilização do LBI na prática do enfermeiro, nos últimos cinco anos foi possível identificar a escassez da produção científica na área. A produção encontrada nas bases de dados nacionais e internacionais são incipientes e apresentam métodos baseados em revisões e poucos estudos clínicos de alto rigor metodológico. Por conseguinte, as evidências produzidas apresentam baixo escore.

Ressalta-se a importância da realização de novos estudos clínicos com métodos que investiguem a exequibilidade e relevância do uso da LBI na prática clínica de enfermagem produzindo evidências capazes de contribuir com a base para uma prática segura e eficaz.

\section{Referências}

Almeida, N., Rodas, S., \& Marques, W. (2020). Investimento em pesquisa e inovação tecnológica: um estudo de caso para o Brasil. Revista Estudo \& Debate, 27(1), 7-28. http://dx.doi.org/10.22410/issn.1983-036X.v27i1a2020.2195

Armelin, M. V. A. L., Jurado, S. R., Saraiva, K. V. de O., Corazza, A. V., Silva, G. D. da, \& Sanchez, A. (2019). O uso do laser de baixa potência por enfermeiro no tratamento de lesões cutâneas e orais. Nursing (São Paulo), 22(253), 3006-3010. https://doi.org/10.36489/nursing.2019v22i253p3006-3010

Bavaresco, T., Osmarin, V., Pires, A., Moraes, V., \& Lucena, A. (2019). Terapia a laser de baixa potência na cicatrização de feridas. Revista de Enfermagem UFPE on line, 13(1), 216-226. https://doi.org/10.5205/1981-8963-v13i1a235938p216-226-2019

Bernardes, L. O., \& Jurado, S. R. (2018) Efeitos da laserterapia no tratamento de lesões por pressão: uma revisão sistemática. Rev Cuid, 9 (3), 2423 - 34. http://dx.doi.org/10.15649/cuidarte.v9i3.574.

Botelho, L. L. R., Cunha, C. C. de A., \& Macedo, M. (2011). O método da revisão integrativa nos estudos organizacionais. Gestão E Sociedade, 5(11), 121136. https://doi.org/10.21171/ges.v5i11.1220

Busatta, B. B., Medeiro, K. C., Velozo, L.R., Kakihata, C.M.M., Soares, F. dos S., Azevedo, M. R. B., \& Bertolini, G. R. F. (2018). Uso da terapia a laser de baixa intensidade em estrias distensas: um ensaio clínico randomizado. Scientia Medica, 28 (2), ID28710. https://doi.org/10.15448/1980-6108.2018.2.28710

Brandão, M. G. S., Moreira Ximenes, M. A., Ramalho, A., Veras, V., Barros, L., \& Araujo, T. (2020). Efeitos da laserterapia de baixa intensidade na cicatrização de úlceras nos pés em pessoas com diabetes mellitus. ESTIMA, Brazilian Journal of Enterostomal Therapy, 18. https://doi.org/10.30886/estima.v18.844_PT

Castro, M. F. de, Barbosa, L. R. P., \& Silva, L. L. da. (2020). Ação da terapia a laser de baixa intensidade na cicatrização de ulcerações diabéticas. Research, Society and Development, 9(10), e6239109109-e6239109109. https://doi.org/10.33448/rsd-v9i10.9109

Conselho Regional de Enfermagem de São Paulo (2014). Parecer COREN-SP 009/2014 - CT: Utilização do Laser de Baixa Intensidade (LBI) pelo enfermeiro. COREN-SP.

Danski, M. T. R., Oliveira, G. L. R. de, Pedrolo, E., Lind, J., \& Johann, D. A. (2017). Importância da prática baseada em evidências nos processos de trabalho do enfermeiro / Importance of evidence-based practice in nurse's work processes <b\&gt;. Ciência, Cuidado E Saúde, 16(2). https://doi.org/10.4025/cienccuidsaude.v16i2.36304 
Gomes, C. F., \& Schapochnik, A. (2017) O uso terapêutico do laser de baixa intensidade (LBI) em algumas patologias e sua relação com a atuação na fonoaudiologia. Distúrb Comum, 29 (3), 570-578. http://dx.doi.org/10.23925/2176-2724.2017v29i3p570-578

Henriques, Á. C. G., Cazal, C., \& Castro, J. F. L. de. (2010) Ação da laserterapia no processo de proliferação e diferenciação celular: Revisão da literatura. Revista do Colégio Brasileiro de Cirurgiões, 37, 295-302. https://doi.org/10.1590/S0100-69912010000400011

Lima, N. E. P., Gomes, G. de M., Feitosa, A. do N. A., Bezerra, A. L. D., \& Sousa, M. N. A. de. (2018). Laser therapy low intensity in wound care and practice nurses / Laserterapia de baixa intensidade no tratamento de feridas e a atuação da enfermagem / Terapia de láser de baja intensidad en el tratamiento de heridas. Revista de Enfermagem da UFPI, 7(1), 50-56. https://doi.org/10.26694/2238-7234.7150-56

Lino, M. M., Backes, V. M. S., Costa, M. A. da S. M. C. da, Martins, M. M. F. P. da S., \& Lino, M. M. (2017). Influências capitalistas na produção do conhecimento em enfermagem. Revista Gaúcha de Enfermagem, 38(1). https://doi.org/10.1590/1983-1447.2017.01.61829

Lopes, L. D., Rodrigues, A. B., Brasil, D. R. M., Moreira, M. M. C., Amaral, J. G., \& Oliveira, P. P. de. (2016). Prevenção e tratamento da mucosite em ambulatório de oncologia: uma construção coletiva. Texto \& Contexto - Enfermagem, 25(1). https://doi.org/10.1590/0104-070720160002060014

Macedo, S. P. R., Mota, M. S.A., Fagundes, C. F., Souza, M. R., \& Navarro, R. S. (2021). Efeitos da fotobiomodulação no tratamento das úlceras por pressão: revisão integrativa. Research, Society and Development, 10 (2), e32810212597. https://doi.org/10.33448/rsd-v10i2.12597

Melnyk B. M., \& Fineout-Overholt, E. (2014) Evidence-based practice in nursing \& healthcare: a guide to best practice. (3a ed.), Lippincott Williams \& Wilkins.

Nassif, M. S., Iunes, D. H., Sousa, L. de, Costa, I. C. P., Oliveira, P. E. de, Moura, C. de C., Menezes, F. da S., Mantuani, A. P. A., \& Chaves, E. de L. (2020). Validação de um protocolo de auriculoterapia com laser para dor crônica na coluna vertebral. Revista Mineira de Enfermagem, 24(0), 1-9. https://doi.org/10.5935/1415.2762.20200087

Oliveira, A. C. de., Abreu, B. M. ., Cavalcante , S. B. .., \& Silva, W. F. . (2021). Efficacy of low power laser therapy in diabetic ulcers . Research, Society and Development, 10(13), e569101321608. https://doi.org/10.33448/rsd-v10i13.21608

Oliveira, D. M., Costa, M. M. L., \& Malagutti, W. (2019). Intervenções de enfermagem para pacientes com lesão por pressão. Revista de Enfermagem UFPE on line, 13(0), Article 0. https://doi.org/10.5205/1981-8963.2019.240237

Pedrolo, E., Danski, M., Mingorance, P., de Lazzari, L., Méier, M., \& Crozeta, K. (2009). A prática baseada em evidências como ferramenta para prática profissional do enfermeiro. Cogitare Enfermagem, 14(4). http://dx.doi.org/10.5380/ce.v14i4.16396

Pereira, C. D., Alvim, N. A. T., Pereira, R. D. de M., Bergold, L. B., Junior, S. C. dos S. G., \& Bastos, V. D. (2021). Laser-acupuntura no controle da glicemia na Diabetes tipo II: Ensaio clínico randomizado. Enfermería Global, 20(2), 492-530. https://doi.org/10.6018/eglobal.443241

Pereira, R. D. de M., Alvim, N. A. T., Pereira, C. D., \& Gomes Junior, S. C. dos S. (2018). Protocolo de laser-acupuntura para hipertensão arterial sistêmica primária: Ensaio clínico randomizado. Revista Latino-Americana de Enfermagem, 26. https://doi.org/10.1590/1518-8345.1887.2936

Persilva, M. (2019). Laserterapia de baixa intensidade no tratamento adjuvante em lesões cutâneas: Uma revisão bibliográfica. Revista Feridas, 36, $1241-1248$.

Pérez Júnior, E. F.; Pires, A. da S.; Monteiro, M. J.; Moraes, K. de.; Santos, L. D.; Benevides, J. M. M.; Coutinho, V. L., \& Gonçalves, F. G. de A. (2021) Lowlevel laser therapy: Characteristics of clients treated at the Clinical Podiatrics servisse. Research, Society and Development, $10(4)$, e36610414099. https://doi.org/10.33448/rsd-v10i4.14099

Pires, A. da S., Júnior, E. F. P., Marques, E. G., Thuler, S. R., Jacob, V. L. L., Gonçalves, F. G. de A., Tavares, K. F. A., Santos, J. A., Coutinho, V. L., Peres, E. M., \& Nunes, A. S. A. (2021). Implementação do serviço de enfermagem em Podiatria Clínica em unidade pública de saúde ambulatorial. Research, Society and Development, 10(6), e2710615353-e2710615353. https://doi.org/10.33448/rsd-v10i6.15353

Reichembach, M. T., \& Pontes, L. (2020). Pesquisas inovadoras na enfermagem: Uma mudança necessária. Revista Brasileira de Enfermagem, 73(4). https://doi.org/10.1590/0034-7167-2020730401

Rodrigues, J. M. S., Acosta, A. da S., Gouvea, P. B., \& Massaroli, R. (2020). Uso do laser de baixa intensidade nas radiodermites: Revisão sistemática / Low intensity laser use in radiotherapy lesions: systematic review. Journal of Nursing and Health, 10(2), Article 2. https://doi.org/10.15210/jonah.v10i2.17831

Schmidt, M. H., \& Pereira, A. D. (2016). Laserterapia: A utilização da tecnologia na intervenção em enfermagem. Disciplinarum Scientia | Saúde, 17(3), 499506.

Tallamini, I., \& Pinheiro S. M. L. (2020) Processo de cicatrização e efeito da laserterapia de baixa potência: revisão integrativa. Revista Ciência \&Amp; Humanização Do Hospital De Clínicas De Passo Fundo, 1(1), 123-137. 